\title{
ESTIMATION OF THE INTERNAL EXTINCTION OF SPIRAL GALAXIES FOR MULTI-COLOR TULLY-FISHER
}

\section{RELATIONS}

\author{
MASARU WATANABE \\ National Astronomial Observatory of Japan \\ Osawa 2-21-1, Mitaka, Tokyo 181, Japan \\ AND \\ NAOKI YASUDA \\ University of Tokyo \\ Yayoi 2-11-16, Bunkyo-ku, Tokyo 113, Japan
}

We calculate $B$-, $R$ - and $I$-band internal extinction $A_{i}^{(\lambda)}$ (absorption + scattering) for spirals consisting of an exponential dust layer, a stellar disk and a bulge. The result is applied to local calibrators and cluster spirals (Virgo and Ursa Major) to examine whether or not the wavelength dependence of the relative zero point difference of Tully-Fisher (TF) relations between for local calibrators and for cluster spirals (Pierce \& Tully, 1992) could be accounted for by a variation of $A_{i}^{(\lambda)}$ on the optical depth of galaxies. The extinction is calculated using Monte-Carlo simulations prescribed by Bianchi et al. (1996). For the extinction curve we adopted the one of Cardelli et al. (1989). It is found that a differential extinction $A_{i}^{(R \text { or } I)}-A_{i}^{(B)}$ as a function of the optical depth $\sigma^{(B)}$ has finite upper limits of $\sim 0.3-0.5 \mathrm{mag}$, depending on an inclination of the spiral. These limits are generally smaller than the offset of the TF relative zero point difference. This indicates that the offset may be fully due to an intrinsic color difference between local calibrators and cluster galaxies, or else that the current extinction model is yet to realize a practical extinction process or geometrical configuration of spirals.

\section{References}

Bianchi, S., Ferrara, A., \& Giovanardi, C. (1996) Ap.J., Vol. 465, pp. 127-144

Cardelli, J.A., Clayton,G.C., \& Mathis, J.S. (1989) Ap.J., Vol. 345, pp. 245-256

Pierce, M.J., \& Tully, R.B. (1992) Ap.J., Vol. 387, pp. 47-55 